\title{
Comparison of Laryngeal Mask Airway (LMA) and Endotracheal Tube (ETT) Placement in Neonates
}

Amanda Wanous ${ }^{1}$, Andrew Wey ${ }^{2}$, Kyle Rudser ${ }^{2}$, Kari Roberts ${ }^{3}$

${ }^{1}$ University of Minnesota, Minneapolis, MN; ${ }^{2}$ Division of Biostatistics, University of Minnesota, Minneapolis, MN;

${ }^{3}$ Department of Pediatrics, University of Minnesota Masonic Children's Hospital, Minneapolis, MN

\section{Background and Objective}

With increased use of non-invasive ventilation, there has been an increased focus of research on less invasive methods of delivering surfactant to neonates with respiratory distress syndrome. We investigated the use of a laryngeal mask airway (LMA) for surfactant administration for infants requiring continuous positive airway pressure (CPAP). Infants who met treatment failure criteria in the LMA and Control groups were intubated and given surfactant via an endotracheal tube (ETT).

This study compares placement of an LMA to placement of an ETT in neonates.

\section{Methods}

This is part of a multicenter, randomized controlled trial.

Eligibility Criteria:

- 28 - 36 weeks

- Weight $\geq 1250 \mathrm{~g}$

- Age $\leq 36$ hours old

- On nCPAP with FiO2 30-40\%

- CXR and clinical signs of RDS

Infants were randomized into:

LMA Group: surfactant given via an LMA, placed back on CPAP

Control Group: remained on CPAP, no surfactant given

Intubation criteria was the same for both groups

Videotape of LMA $(n=36)$ and ETT $(n=31)$ placement were reviewed to determine the time and number of attempts required for successful placement. Heart rate (HR) and oxygen saturation ( $\mathrm{SaO} 2)$ change from baseline were analyzed as measures of physiologic stability during placement.
Results: Subject Characteristics

\begin{tabular}{|c|c|c|}
\hline & $\begin{array}{c}\text { LMA } \\
(n=36)\end{array}$ & $\begin{array}{c}\text { ETT } \\
(n=31)\end{array}$ \\
\hline $\begin{array}{c}\text { Birth } \\
\text { Weight, } \\
\text { mean } \pm S D \\
\text { (range), g }\end{array}$ & $\begin{array}{c}2007 \\
\pm \quad 483 \\
(1290- \\
3180)\end{array}$ & $\begin{array}{c}2059 \\
\pm 531 \\
(1254- \\
3305)\end{array}$ \\
\hline $\begin{array}{c}\text { Gestational } \\
\text { age, mean } \\
\pm \mathrm{SD} \\
\text { (range), } \\
\text { wks }\end{array}$ & $\begin{array}{c}326 / 7 \\
\pm 15 / 7 \\
(293 / 7 \\
-354 / 7)\end{array}$ & $\begin{array}{c}324 / 7 \\
\pm 16 / 7 \\
(285 / 7 \\
-356 / 7)\end{array}$ \\
\hline
\end{tabular}

Results: Time and Number of Attempts

\begin{tabular}{|c|c|c|c|}
\hline & $\begin{array}{c}\text { LMA } \\
(n=36)\end{array}$ & $\begin{array}{c}\text { ETT } \\
(n=31)\end{array}$ & $\begin{array}{c}\text { p- } \\
\text { value }\end{array}$ \\
\hline $\begin{array}{l}\text { Duration of } \\
\text { Attempt, } \\
\text { mean } \\
\pm S D \\
\text { (range), } \\
\text { sec } \\
\text { median }\end{array}$ & $\begin{array}{c}32 \pm 19 \\
(21-81) \\
28\end{array}$ & $\begin{array}{c}66 \pm 48 \\
(12-171) \\
45\end{array}$ & $<.001$ \\
\hline $\begin{array}{l}\text { Procedure } \\
\text { time, } \\
\text { mean } \pm \\
\text { SD } \\
\text { (range), } \\
\text { sec } \\
\text { median }\end{array}$ & $\begin{array}{c}88 \\
\pm 136 \\
(12-500) \\
30\end{array}$ & $\begin{array}{c}153 \\
\pm 180 \\
(12-627) \\
49\end{array}$ & 0.065 \\
\hline $\begin{array}{l}\text { Successful } \\
<35 \mathrm{sec} \\
<45 \mathrm{sec} \\
<60 \mathrm{sec}\end{array}$ & $\begin{array}{l}64 \% \\
72 \% \\
75 \%\end{array}$ & $\begin{array}{l}61 \% \\
76 \% \\
92 \%\end{array}$ & \\
\hline $\begin{array}{l}\text { Number of } \\
\text { attempts, } \\
\text { mean }\end{array}$ & 1.5 & 1.9 & 0.106 \\
\hline $\begin{array}{l}\text { Successful } \\
1 \text { attempt } \\
2 \text { or more }\end{array}$ & $\begin{array}{l}69 \% \\
83 \%\end{array}$ & $\begin{array}{l}58 \% \\
68 \%\end{array}$ & \\
\hline
\end{tabular}

Results: Physiologic Stability

\begin{tabular}{|c|c|c|c|}
\hline & $\begin{array}{l}\text { LMA } \\
n=36\end{array}$ & $\begin{array}{c}\text { ETT } \\
n=31\end{array}$ & p-value \\
\hline $\begin{array}{c}\text { Heart } \\
\text { Rate, } \\
\text { mean } \\
\pm S D \\
\text { (range), } \\
\text { bpm } \\
\text { median }\end{array}$ & $\begin{array}{c}1 \\
\pm 5 \\
(-9-11) \\
1\end{array}$ & $\begin{array}{c}-1 \\
\pm 8 \\
(-20-16) \\
1\end{array}$ & 0.333 \\
\hline $\begin{array}{c}\mathrm{SaO} 2, \\
\text { mean } \\
\pm \mathrm{SD} \\
\text { (range), } \\
\% \\
\text { median }\end{array}$ & $\begin{array}{c}-7 \pm 7 \\
(-24-1) \\
-3\end{array}$ & $\begin{array}{c}-4 \pm 11 \\
(-23-20) \\
-2\end{array}$ & 0.361 \\
\hline
\end{tabular}

Summary and Conclusion

Duration of attempts was shorter for LMA compared to ETT placement (32 sec vs $66 \mathrm{sec}$, $p<0.001)$. Mean total procedure time for successful LMA placement was $88 \mathrm{sec}$ compared to $153 \mathrm{sec}$ for ETT $(p=0.065)$. Mean number of attempts for successful placement was fewer for LMA placement (1.5 vs 1.9, $p=0.106)$. Physiologic parameters remained near baseline in LMA and ETT groups with $\mathrm{HR}$ change $+1 \mathrm{bpm}$ and $-1 \mathrm{bpm}(\mathrm{p}=0.333)$ and $\mathrm{SaO} 2$ change $-7 \%$ and $-4 \%(p=0.361)$.

Placement of an LMA is well tolerated and required less time and fewer number of attempts as compared to the traditional method of endotracheal intubation.

\section{Funding and Product Support}

Minnesota Medical Foundation, Children's Hospital Assoc: Children's Minnesota, Clinical and Translational Sciences Institute- Univ of Minnesota, Product donations: Curosurf® by Chiesi USA, Inc, LMAs by LMA North America, PediCaps $\AA$ by Covidien, Neo-Verso $®$ Fluid/ Access Catheters by CareFusion 